\title{
SME-SERVICE NETWORKS FOR COOPERATIVE OPERATION OF ROBOT INSTALLATIONS
}

\author{
Peter ter Horst \\ DemarLaserB.V., peter@demarlaser.nl \\ Gerhard Schreck \\ FraunhoferIPK, gerhard.schreck@ipk:fraunhofer.de \\ Cornelius Willnow \\ FraunhoferIPK, cornelius.willnow@ipk.fraunhofer.de
}

GERMANY

\begin{abstract}
A major obstacle for the introduction of industrial robots in small and medium enterprises (SMEs) is formed by the complexity of the robot systems, and the required expertise and qualified personnel. These high requirements for the companies and their personnel could be reduced, if SMEs that dispose over the required skill could provide them to other SMEs as Internet services. This paper describes working procedures, methods and tools for creating such Internet-based SME-service networks.
\end{abstract}

\section{INTRODUCTION}

When introducing industrial robots, small and medium enterprises (SMEs) are today faced with the full complexity of robot systems. I.e. for small enterprises the introduction of robot installations already forms a high investment. Additionally, this is accompanied by the need for qualified personnel that is able to perform the planning, operation and maintenance tasks for the robot system.

While larger companies can afford specialist departments for planning and performing robot application, smaller companies dispose only over a small number of persons, and often only over a single person, for performing all robot related tasks (IFR 2001). This consequently overtaxes the companies and their personnel. For these reasons, SMEs often hesitate to introduce robots, even if this is urgently required for productivity and quality reasons.

Different SMEs together, however, dispose over the required types of expertise for efficiently operating robot installations. They could mutually support each other by providing the required skills as services. The spatial distance between the companies could be bridged by Internet.

In pursuit of this approach, the EU CRAFT-Project 'Small and Medium Enterprises - Robotics Service Inter-Network' (SME-Rosin) was started in November 2002, with a planned duration of two years (SME-Rosin Consortium, 
2003a). In the project, services and tools for robotics support networks for SMEs are developed.

This paper presents developed company network structures, interaction schemes and support tools for cooperative, balanced integration of automated systems and human involvement.

\section{THE EXAMPLE NETWORK}

The SME-Rosin consortium consists of European SMEs that form the SME-network shown in figure 1. It is used as an example for developing and testing the required services and tools.

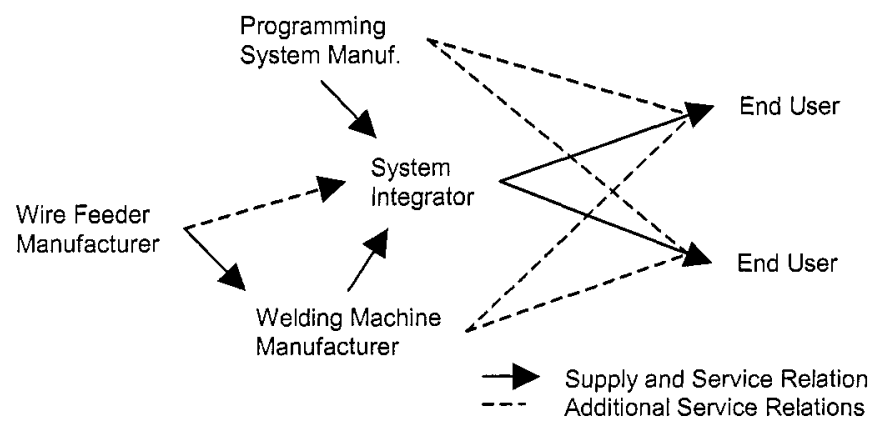

Figure 1 - The example SME-network

The example SME-network includes two typical end user companies. One already disposes over a robot installation and contributes the experience from introducing it and taking it into operation. The other one does not yet dispose over a robot and represents the challenge of introducing a robot.

A supply chain is represented by a system integrator, a manufacturer of welding machines and a manufacturer of wire feeder units. A further supply chain for software tools starts at a programming system manufacturer.

The network shows existing customer/supplier relations. Further services, that are developed in SME-Rosin and that are supported by Internet, enable a closer and more efficient cooperation of the companies.

\section{DEVELOPMENT AND VERIFICATION PROCESS}

The development of the service processes takes place in development-verificationcycles as illustrated in figure 2. After an initial analysis phase, service processes are defined and required tools are realized. The resulting developments are then verified in test scenarios and close-to-reality pilot installations. Based on the obtained feedback, the developed processes and tools are improved. Over-all three cycles are performed. 


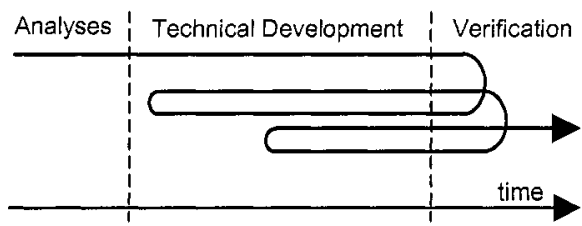

Figure 2 - Development-Verification-Cycles for the service and tools development

\section{SERVICE AREAS}

Based on the analysis performed during the SME-Rosin project, business processes for the service areas illustrated in figure 3 were developed (SME-Rosin Consortium, 2003b).

The area Production Engineering includes services for the development of manufacturing processes for robot cells. This concerns Product Re-design for automation in robot cells, the development of suitable fixtures, and the development of the processing tool and method, e.g. for arc welding.

The service area Programming includes services for Manual and Automatic Programming of robots. The term 'off-line programming' (OLP) denotes the creation of robot programs with simulation models of robot, work cell and work piece. This stands in contrast to 'on-line programming' what denotes programming directly at the physical robot. Programming as a remote service requires off-line programming. The activities for creating and maintaining the required consistency between the real cell and the simulated cell are summarized as Model Consistency.

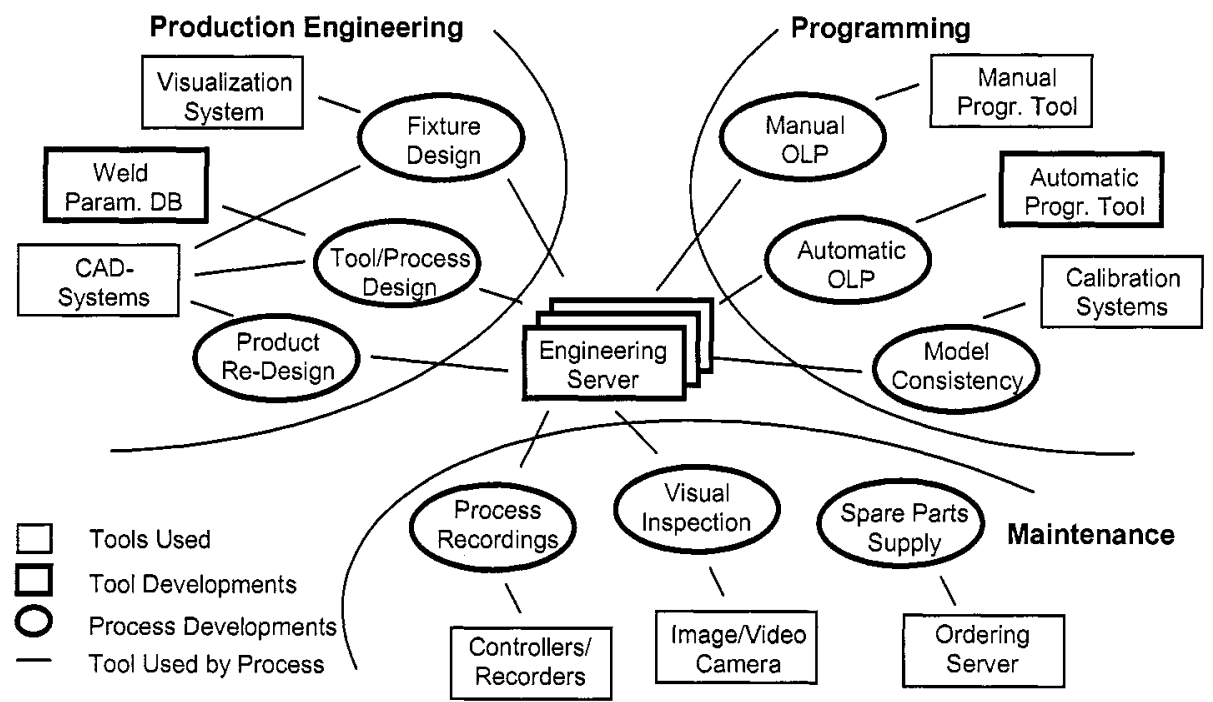

Figure 3 - Service areas, their services and tools involved

The service area Maintenance consists in obtaining Process Recordings for analyzing a robot cell, in Visual Inspection for obtaining pictures and films from a cell and in Spare Parts Supply services. 
In practice, the different services and their business processes are often strongly related to each other. Process Recordings may be required for analyzing process performance during Production Engineering, Production Engineering may require Programming for testing designs, Programming services may be required after spare parts replacement, e.g. for re-establishing Model Consistency.

\section{THE ENGINEERING SERVER}

The central tool for organizing Internet-based services is an Engineering Server, as shown in the centre of figure 3 . It organizes the exchange of documents like CADdrawings, measuring data and robot programs. It provides mechanisms for notification, archiving and project progress control (Berger, Hohwieler 2003).

The SME-Rosin Engineering Server is especially designed for SME-networks. The SMEs are already burdened with the introduction and operation of the robot system. Therefore, the Engineering Server has to be very simple to use and to integrate into existing infrastructures (Kärkkäinen, Ala-Risku, 2003). It has to noticeably facilitate work and, for this reason, its usage has to flexibly fit into actual work procedures and current user needs.

Furthermore, it has to be possible to arbitrarily combine Internet-based activities with conventional means like telephone, fax, e-mail and travelling. Since almost all activities of robot planning and operation are strongly related to physical processes, the service provider has to be well familiar with the cell and has to have a good understanding of the manufacturing process. For a number of activities, direct at-site presence of the service provider is indispensable.

Consequently, activities via Internet have to be executable in combination with conventional means. For this reason, service processes have to be designed in a way that allows to combine conventional means with Internet activities, as required by the actual situation and practical needs. This reflects the flexibility that is a major strength of SMEs.

\section{COOPERATIVE INTERACTION SCHEMES}

In the course of the project, interaction schemes for the service processes described above are developed. As a typical, but concise example, the interaction scheme for Product Re-Design is discussed in more detail.

The Engineering Server is the central means for project organization between the different involved actors, as illustrated in figure 4. It is run by the Service Provider, which is the organization that provides the services.

For providing a Re-Design service, the Engineering Server is operated by a Designer employed at the Service Provider. The Service Provider, has a Company Interface to the Service User which is also an organization.

An employee of the Service User is in charge for cooperation with the Service Provider in a given project. For the case of a Product Re-Design project, this may be the Sales Engineer in charge of the project. The Sales Engineer at the Service User cooperates with the Designer at the Service Provider via the Engineering Server. Of course, as practical needs demand, also other communication means are involved.

The cooperation between the Designer at the Service Provider, and the Sales 
Engineer at the Service User follows the diagram of principle activities in figure 5. It starts with the initiation of the service, followed by a number of service cycles.

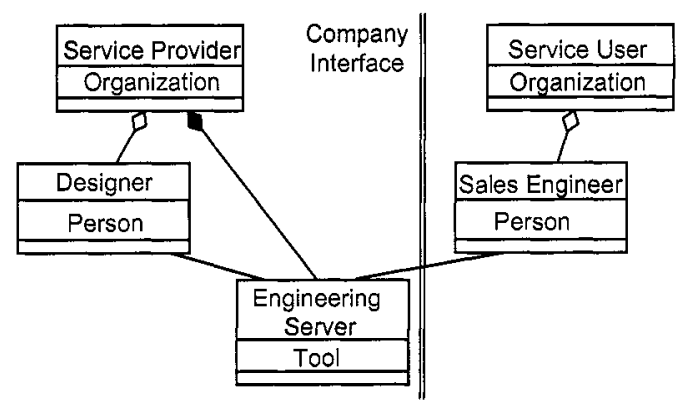

Figure 4-Engineering Server as central means for project coordination

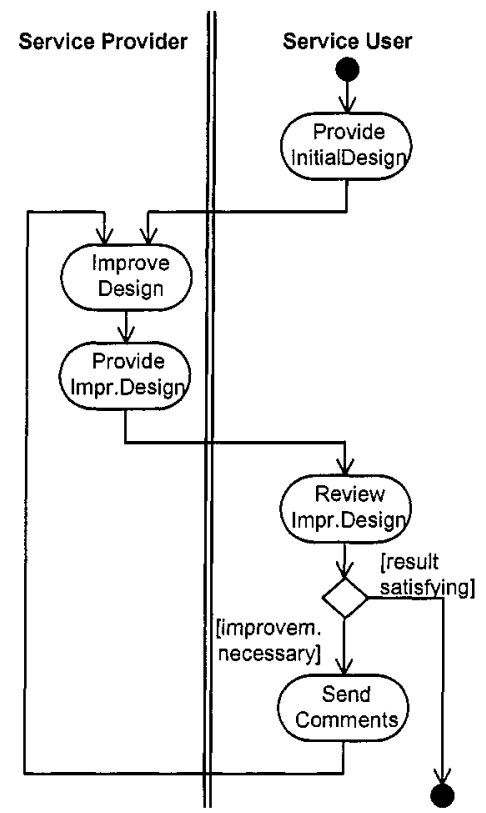

Figure 5 - Basic activity scheme for Re-Design services

After project initiation, the initial design is provided by the Service User and sent to the Service Provider via the Engineering Server. The initial design may consist of CAD-drawings, part lists, material specifications, surface treatments, quality requirements, etc.

For CAD-drawings, standards like Step or Iges could be used. This however may lead to data losses during conversion. Proprietary data formats, e.g. CATIA or AutoCAD files, avoid the losses but need agreements for using the same tool at Service User and Service Provider.

A further relevant technology are graphics viewers. They do not allow to modify data, but allow to inspect designs and they are provided for free. Then Service Providers can use the tool of their choice, while Service Users need no licenses. 
Viewers are also available for simulation and animation tools (Visual 2003).

After providing the initial design, the service cycle starts: The Service Provider reviews and improves the design. During this, further interaction may take place. Additional specifications and requirements can be sent, and details may be clarified by e-mail or telephone. Then the improved design is provided to the Service User. This may include design variants for evaluation and selection by the Service User.

The Service User reviews the improved design. If the result is satisfying, then the Product Re-Design cycle is completed. Otherwise, the Service User comments on the actual version and sends the comments to the Service Provider. The comments may consist of text, modified or commented CAD-drawings, a design review by telephone, etc. as it suits best. For exchange of electronic data, the Engineering Server is used. Then, a new development cycle starts.

Please note again, that this development cycle is nominal and may be altered pragmatically as needed. It may be interrupted by conventional means like telephoning, faxing, travelling, etc. where this is more efficient. The improvement of the design may be interrupted by new data from the Service User. The review of the improved design may be interrupted by new ideas from the Service Provider, etc.

Furthermore this nominal cycle can be combined with the Engineering Processes of other service areas. For an implementation of the cycle in industrial praxis, any need and any degree of flexibility has to be possible.

\section{USE CASE FOR COOPERATIVE PRODUCT RE-DESIGN}

The following use case illustrates the interaction scheme by an example. The example and the names of the involved companies are fictive and used for illustrative purposes only. The example is, however, typical and realistic.

The use case is built on the following scenario: CoMa Ltd. manufactures containers with high volume. The containers have a high number of variants, reaching from differing dimensions to a variety of special equipment like rolls and suspensions. In addition to the number of standard container types, CoMa produces containers also for customer designs. Most of them are derived from standard designs. Sub-assemblies like side parts, bottom parts, back planes and doors are robot welded.

Since the number of customer designs does not justify to employ a skilled designer for automation, CoMa uses services provided by the Re-Design Company $\mathrm{ReDeCo} \mathrm{GmbH}$. ReDeCo disposes over models of the robot installation at CoMa. The cooperation in a project may proceed as followings:

\section{Project Initiation}

1. A customer of CoMa needs containers that are adapted to the customer's existing internal transportation system. For this, containers have to be adjusted in size and the suspension mechanism is to be modified.

2. During a meeting at the customer, hand sketches for the new design are made, and CoMa is supplied with printouts of technical drawings for the suspension and dimensions.

3. The sales engineer at CoMa creates a new AutoCAD design of the customized container variant, by modifying an existing design.

4. ReDeCo $\mathrm{GmbH}$ is contacted by telephone for the new task.

5. CoMa's sales engineer places the new AutoCAD design in a new folder of 
ReDeCo's Engineering Server.

6. The designer in charge at $\mathrm{ReDeCo}$ is notified by the Engineering Server that the new design is available.

\section{First Re-Design Cycle}

7. The designer at ReDeCo fetches the design from the Engineering Server and reviews it. It turns out that the suspensions are placed in a way that the tool collides with them when welding the vertical stiffeners of side parts.

8. With a telephone call, ReDeCo checks with CoMa if it is better to shift the suspensions or to shift the stiffeners. Since the suspensions have to fit to the customers's transportation system, they may not be shifted.

9. Since shifting the stiffeners may reduce the admissible load of the containers, CoMa checks with the customer for the maximal load. The result it sent to ReDeCo via the Engineering Server.

10. The designer at $\mathrm{ReDeCo}$ is notified that the feed back arrived and fetches it from the Engineering Server.

11. The designer at ReDeCo shifts the stiffeners in the AutoCAD design. The admissible payload is verified by a tool for stress analysis. The resulting design is placed in the Engineering Server.

12. The sales engineer at CoMa is notified and fetches the new design from the Engineering Server. It is checked for compliance with the customer's specification.

\section{Second Re-Design Cycle}

13. The new distribution of stiffeners in combination with the reduced payload suggests to omit stiffeners. This would reduce production costs. CoMa creates a re-design and passes it via the Engineering Server to ReDeCo.

14. The designer at ReDeCo is notified about the feed back and clarifies the reasons for the modifications during a telephone conversation.

15. ReDeCo reviews the design, tests it with the tool for stress analysis and returns it with minor modifications.

16. CoMa reviews the redesign with the customer, who accepts it.

17. The acceptance is sent via the Engineering Server to ReDeCo who uses the data in the Engineering Server for accounting and places the invoice.

18. CoMa passes the design to production, where the robot programs are created (this could be done by a Programming Service Provider).

19. The containers are produced.

\section{BUSINESS ADVANTAGES}

The central benefit from the developed interaction schemes for Internet services is that robot end user companies are relieved from various tasks in robot application and from the need for highly skilled and specialized personnel. This holds for all service processes of the described service areas.

The given example use case for Product Re-Design services shows that CoMa does not need to employ an experienced product re-designer, CoMa can profit from the experience of ReDeCo in Product Re-Design, and CoMa can benefit from the specialised tools that ReDeCo disposes over, e.g. for computing stress models.

The advantage of ReDeCo is an extended market. Re-Design services can be offered also to companies that had no need for them. The efficient cooperation of both companies is enabled by defined interaction schemes and data exchange mechanism that are well known to the partners. This is supported by the Engineering Server that organises data exchange and project progress control. 


\section{SUMMARY}

The introduction of robot installations at small and medium enterprises (SME) end user companies is faced with the challenge of the complexity of handling robot systems. This requires expertise and a high educational level of the personnel. For facilitating the introduction of robots in SMEs, the EU project 'Small and Medium Enterprises - Robotics Service Inter-Network' (SME-Rosin) was started. It aims at developing Internet-based services that support the planning, operation and maintenance of robots at SMEs by services of SMEs that dispose over the required special expertise.

In the project, services for the areas Production Engineering, Programming and Maintenance are developed. Each service area defines a number of interaction schemes for performing the required distributed cooperation of SMEs. The cooperation schemes flexibly integrate company interaction via Internet with conventional means like telephoning and traveling.

As the central means for project coordination and data exchange, an Engineering Server is developed. It is especially tailored for the needs of SME-networks. It is easy to use and its operation flexibly adapts to current project needs. This reflects the flexibility that forms a typical strength of SMEs.

The cooperation schemes for the services and the required tools are developed and verified repeatedly in a number of development-verification-cycles.

The resulting service concepts were illustrated in this article by interaction schemes for Product Re-design, followed by a close-to-reality use case.

Acknowledgements. The project SME-Rosin (Small and Medium Enterprises Robotics Service Inter-Network) is funded by the European Commission as FP-5 CRAFT Project IST 2001-55039. The consortium is formed by Industrieausstatter fuer Schweissen und Umwelttechnik GmbH (ISU), Neubrandenburg Germany, Visual Components Oy (Helsinki Finnland), Cooptim Ltd., (Erd (Budapest), Hungary), rs-technologies (Leipzig, Germany), Demar Laser B.V. (Hengelo, The Netherlands), MEBIA GmbH (Ossendorf, Germany) Fraunhofer IPK (Berlin, Germany), ZDIS (Gliwice, Poland).

\section{REFERENCES}

1. IFR International Federation of Robotics, "European Robotics, A white paper on the status and opportunities of the European Robotics Industry", Prepared by European Robotics Forum (IFR ERF) \& European Robotics Research Network (EURON), September 2001

2. SME-Rosin Consortium (a), SME-Rosin Homepage, http://sme-rosin.ipk.fhg.de/, updated continuously

3. SME-Rosin Consortium (b), "Infra Structure: Distributed Work Procedures", project documentation, 4 December 2003, publication planned

4. Berger R., Hohwieler E., "Service Platform for Web-based Services for Production Systems", Proceeding of the 36th CIRP International Seminar on Manufacturing Systems, June 03-05, 2003, Saarland University Saarbrücken, Germany

5 Mikko Kärkkäinen, Timo Ala-Risku, "Facilitating the Integration of SMEs to Supply Networks with Lean IT Solutions", Building the Knowledge Economy: Issues, Applications, Case Studies, IOS Press, 2003

6. Visual Components Oy, "3DCreate (White Paper)", http://download.visualcomponents.net/docs/VC_whitepaper.pdf 